\title{
Data Synchronization in Operando Gas and Heating TEM
}

Fan Zhang ${ }^{1,3}$, Merijn Pen ${ }^{2}$, Ronald G.Spruit ${ }^{2}$, Hugo Perez Garza ${ }^{2}$, Wei Liu $^{1 *}$, Dan Zhou ${ }^{2 *}$

1 Dalian National Laboratory for Clean Energy, Dalian Institute of Chemical Physics (DICP), Chinese Academy of Sciences, Dalian, China. 2 DENSsolutions B.V., Delft 2628 ZD, The Netherlands

3 University of Chinese Academy of Sciences, Beijing, China.

Corresponding author:

dan.zhou@denssolutions.com

weiliu@dicp.ac.cn

Highlights

Functional relationships are drawn between the delayed time and the NanoReactor flow rate and pressure.

Automatic data synchronization in operando TEM scripts are developed and released in open source.

\begin{abstract}
Time-resolved correlations between the environment, the reaction products, the energy transfer and the material structures during the reaction processes make operando gas and heating TEM more and more attractive in recent years. The intrinsic time delays that exist among parameter measurement locations need to be calibrated for valid correlations. Otherwise, erroneous conclusions would be drawn, such as overestimating the critical temperatures, or mismatching the structure and composition relationships to activities. Herein, we report on a method measuring and calibrating the time delays involved in operando TEM. This method relies on the unique capability of on-chip calorimetry of the gas Nano-Reactor. It is shown that the time delay depends on the gas flow rate and pressure, and has little dependence on the gas type. A functional relationship fitted between the time delay and the gas flow rate can automize the time delay calibration and thus synchronize the data from different locations. Based on the investigations, we developed algorithms and scripts to enable the automatic data synchronization in operando gas and heating TEM in both real time experiments and
\end{abstract}


post experiments.

Keywords

\section{Data synchronization; Operando TEM; Time delay; Automatic characterization and calibration}

\section{Introduction}

To develop high efficient, eco-friendly and cost wise catalyst materials, disclosing the reaction dynamics from micro- to atomic- scale is critically important (1-3). Catalysts normally undergo dynamic changes during operation, i.e. their structure and reactivity are coupled to the detailed reaction environmental parameters (4-6). The conclusions made from the ex-situ transmission electron microscope (TEM) characterizations, which compares the pre- and post-reaction catalyst structure and composition, would be insufficient and misleading.

For gas-solid reactions, both the differential pumping system enabled environmental TEM (ETEM) (7) and the microelectromechanical systems (MEMS)based closed-cell TEM holder (8) have made it possible to characterize the materials under the industrially relevant gas and thermal or electrical conditions with TEM's intrinsic advantage of high spatial resolution (9-12). The capability to quantitatively detect the reaction products simultaneously with microstructure observation, is of crucial significance in correlating the catalyst's structure evolution with the catalytic performance and identifying the active sites and catalytic mechanisms (13-16). This brought the TEM characterization from the in-situ scenario to the operando scenario $(17,18)$.

For example, Vendelbo et al. made use of the operando TEM and reported the synchronous observations of oscillating $\mathrm{CO}$ oxidation rate and periodic refracting of its catalyst Pt nanoparticles. Although more complexity could contribute to the reactions, this finding brought direct evidence and insight to the correlations between catalytic reactions and catalyst nanoparticle shape dynamics (19). Tan et al. reported that the $\mathrm{Pt}-$ Ni rhombic dodecahedron NPs with segregated $\mathrm{Pt}$ frames evolve into a yolk $(\mathrm{Pt})-$ shell (NiO) structure when catalyzing $\mathrm{CO}$ oxidation, whereas the more roundish Pt-Ni alloy NPs tend to separate into a two-sided structure with $\mathrm{Pt}$ and $\mathrm{NiO}$ on each side. These observations suggest that the initial architecture of catalysts can determine their structural evolution in the reactions, and thus provide fundamental basis for catalysts design and optimization (20). Milivoj et al. investigated the chemical dynamics of CO 
oxidation over Pt NPs in the entire range of catalytic conversion by operando TEM. They found that chemical dynamics consist of morphological transformations at the low activity regime and fluctuating structural dynamics at the high activity regime. They also observed that shape dynamics could contribute to oscillating CO oxidation rate in high activity regime (similar to what Vandelbo et al. reported), and to catalyst deactivation during catalytic cycling (as revealed by higher and higher ignition temperature). They demonstrated how powerful the operando TEM is in building the temporally and spatially resolved multiple factor correlations in a complete reaction process (21). In all these works, one important, while could be ignored or taken for granted point is the time delays between different parameter measurement locations. To simplify the explanation of the time delays, a schematic view of the operando set-up is shown in Figure 1(a) $(22,23)$. As shown in Figure 1(a), taking a MEMS-based NanoReactor Operando TEM set-up as an example, the gas needs to travel from a Gas Supply System (GSS) into the TEM, and then from the TEM to the MS. The initial gas composition will be measured in the GSS while the outlet gas will be measured in the MS. As shown in Figure 1(b), a user set gas composition change in the GSS will show changes in the MS after $79.1 \mathrm{~s}$. That is to say, simultaneously measured data don't naturally mean they are synchronized.

Different from the GSS using flow meters and the MS using the ionized gases' mass to charge ratio, there are no direct gas composition measurements made inside the TEM Nano-Reactors, which is the area of the reaction occurring and the target of TEM investigations. A parameter inside the TEM Nano-Reactor e.g. gas composition in this paper, is needed to synchronize all the other data to TEM observations.

Former researches manually calibrated the time-delay case by case $(16,19-21)$, which is relatively low efficiency and non-applicable to experiments with varying gas environment during reaction. 
a

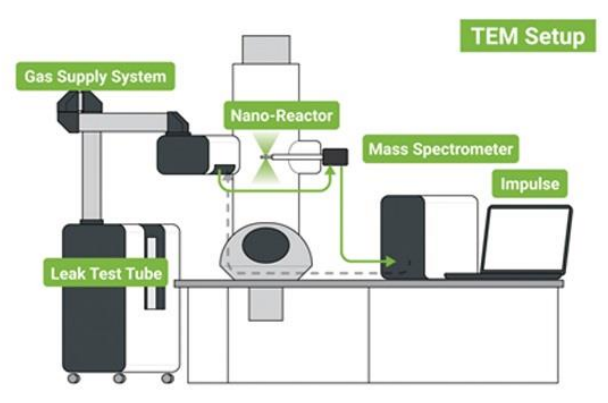

b

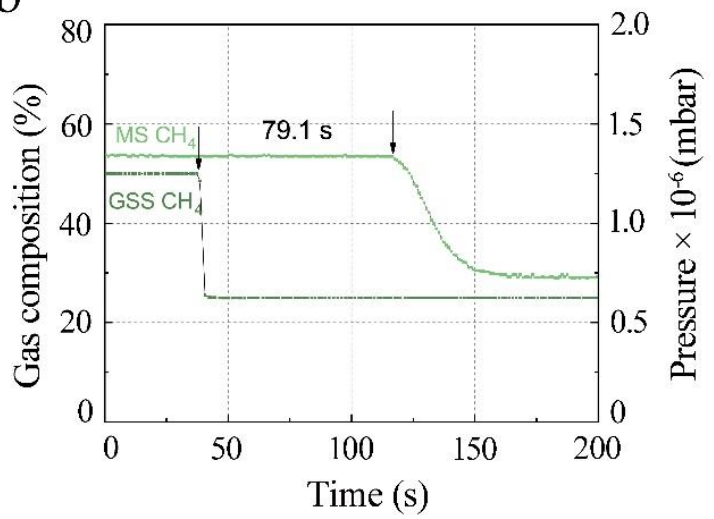

Figure 1. (a) A schematic view of a gas cell based operando TEM gas path; (b) Illustration of time-delay between GSS and MS. Here GSS data are measured by flow meter (24) and MS by measuring the ionized gases' mass to charge ratio (25).

In this work, we will present a data synchronization, i.e. time delay calibration method in operando TEM studies enabled by the nano-calorimetry localized on TEM Nano-Reactors. The relationship of the delayed time to reaction conditions, e.g. the gas pressure, flow rate, and gas composition were investigated systematically. Afterwards, open source codes have been developed to achieve reliable and automated data synchronization with time-delay characterization on-line and time-delay calibration off-line. Finally, we propose a general protocol to perform automatic time delay calibration for their own operando TEM set-ups, which could have differences case to case. The situation of an ETEM is more complicated than the gas cell. It is out of scope of the current manuscript.

\section{Materials and Instruments}

The experimental operando TEM set-up used the DENSsolutions Climate Gas Supply System (GSS), Climate in-situ TEM gas and heating sample holder in a Thermo Scientific Themis ETEM and Climate in-situ gas analyzer. The gases $\mathrm{H}_{2}, \mathrm{CH}_{4}, \mathrm{O}_{2}, \mathrm{CO}_{2}$ of $99.999 \%$ or higher purity are used as purchased. An empty Nano-Reactor, without any samples on it, was used for systematic investigations.

\section{Operando Gas and Heating TEM Set-ups}

Complementary to Figure 1(a), a diagram of the operando gas and heating TEM set-ups used in current work is shown in Figure 2. From the hardware perspective, this set-up can be grouped to GSS, TEM and MS, as shown in Figure 1. Following the gas path and from TEM investigation perspective, this set-up can be divided into pre-TEM, in-TEM and post-TEM. 
Pre-TEM Three gas bottles are connected to the GSS, which contains three flow controllers that measure and regulate their flow rates $\left(F_{\text {gas } 1}, F_{\text {gas } 2}, F_{\text {gas } 3}\right)$. Their relative ratios determine the gas composition of the final mixture. The mixture is made by merging the gas flows into a specially designed low-volume mixing valve where they can be mixed without interrupting the continuity of the flow and therefore the continuity of the gas experiment. As a result of this on-the-fly mixing, the composition of the gas mixture can be adjusted to the desired composition within very short response times. In addition, the multi-functional mixing valve can guide the gases either to the NanoReactor, or directly to the exhaust. This allows for very steep and abrupt changes of gas composition, and provides a very fast and flexible method in controlling the ratio of the three gases, i.e. the gas composition that is guided into the Nano-Reactor and interacts with the sample.

Pre-TEM parameters include three gas flow rates $\left(\mathrm{F}_{\text {gas } 1}, \mathrm{~F}_{\text {gas } 2}, \mathrm{~F}_{\text {gas } 3}\right)$.

In-TEM The mixed gas will be guided to the TEM holder and modulated by an inlet and an outlet pressure $\left(\mathrm{P}_{\text {in }}\right.$ and $\left.\mathrm{P}_{\text {out }}\right)$ to meet the Nano-Reactor gas pressure and flow rate requirement inside the Nano-Reactor, following these two equations:

$$
\begin{aligned}
& P_{N R}=\sqrt{A P_{\text {in }}^{2}+B P_{\text {out }}^{2}} \\
& F_{N R} \propto\left(P_{\text {in }}-P_{\text {out }}\right)(2)
\end{aligned}
$$

If equal distances of $P_{N R}$ to $P_{\text {in }}$ and $\mathrm{P}_{\text {out }}$ controllers are assumed in Eq.(1), $\mathrm{A}=\mathrm{B}=0.5$ will be applied, as used by DENSsolutions softwares as default values; if the distances are not equal, different values will be applied with $\mathrm{A}+\mathrm{B}=1, \mathrm{~A}$ equals the ratio of $P_{N R}$ and $\mathrm{P}_{\text {out }}$ distance to $\mathrm{P}_{\text {in }}$ and $\mathrm{P}_{\text {out }}$ distance, and $\mathrm{B}$ equals the ratio of $\mathrm{P}_{\text {in }}$ and $P_{N R}$ distance to $\mathrm{P}_{\text {in }}$ and $\mathrm{P}_{\text {out }}$ distance. As the flow rate also depends on other parameters, like connecting tubing diameter, Nano-Reactor design and so on, only a proportional relationship is presented here. Although $\mathrm{P}_{\text {in }}$ and $\mathrm{P}_{\text {out }}$ controllers are physically located in GSS (pre-TEM), their values give the gas pressure and flow rate inside Nano-Reactor without time-delay, as is revealed by these two equations. Therefore, $\mathrm{P}_{\text {in }}$ and $\mathrm{P}_{\text {out }}$ are grouped into in-TEM parameters.

The TEM holder provides the platform for connecting the GSS to the MEMSbased gas and heating Nano-Reactor composed of a top chip, O-ring and bottom chip with microheater. The TEM sample can be prepared onto the microheater area of the Nano-Reactor by drop casting, vapor deposition or focused ion beam cutting and thinning. A heating control unit located inside the GSS is used to measure and regulate 
the local temperature of the sample through a closed feedback loop between temperature and heating power, as discussed more in detail in section 4.1. Therefore, the in-TEM parameters include the temperature (T), heating power $(\mathrm{P})$, gas composition, inlet pressure $\left(\mathrm{P}_{\text {in }}\right)$, outlet pressure $\left(\mathrm{P}_{\text {out }}\right)$, gas pressure $\left(P_{N R}\right)$ and gas flow rate $\left(F_{N R}\right)$ in the Nano-Reactor. Please be aware that, the gas composition here, in unit of percentage (of total gas volume), can be different from their values in Pre-TEM part.

Post-TEM In the operando TEM set-up, the gas leaving the TEM holder partially goes to the gas analyzer and partially goes to GSS for outlet pressure control (of which the importance has been shown in Eq.(1) and Eq.(2). The gas analyzer used is a TEMoptimized mass spectrometer, which requires a very small amount of the gas flow to determine the gas composition. This ensures nearly all the gas flows into the GSS for more reliable outlet pressure control. It can analyze the reaction precursor and product gas composition along with TEM investigations. So, the post-TEM parameters include the partial pressures of precursors and products measured by MS.

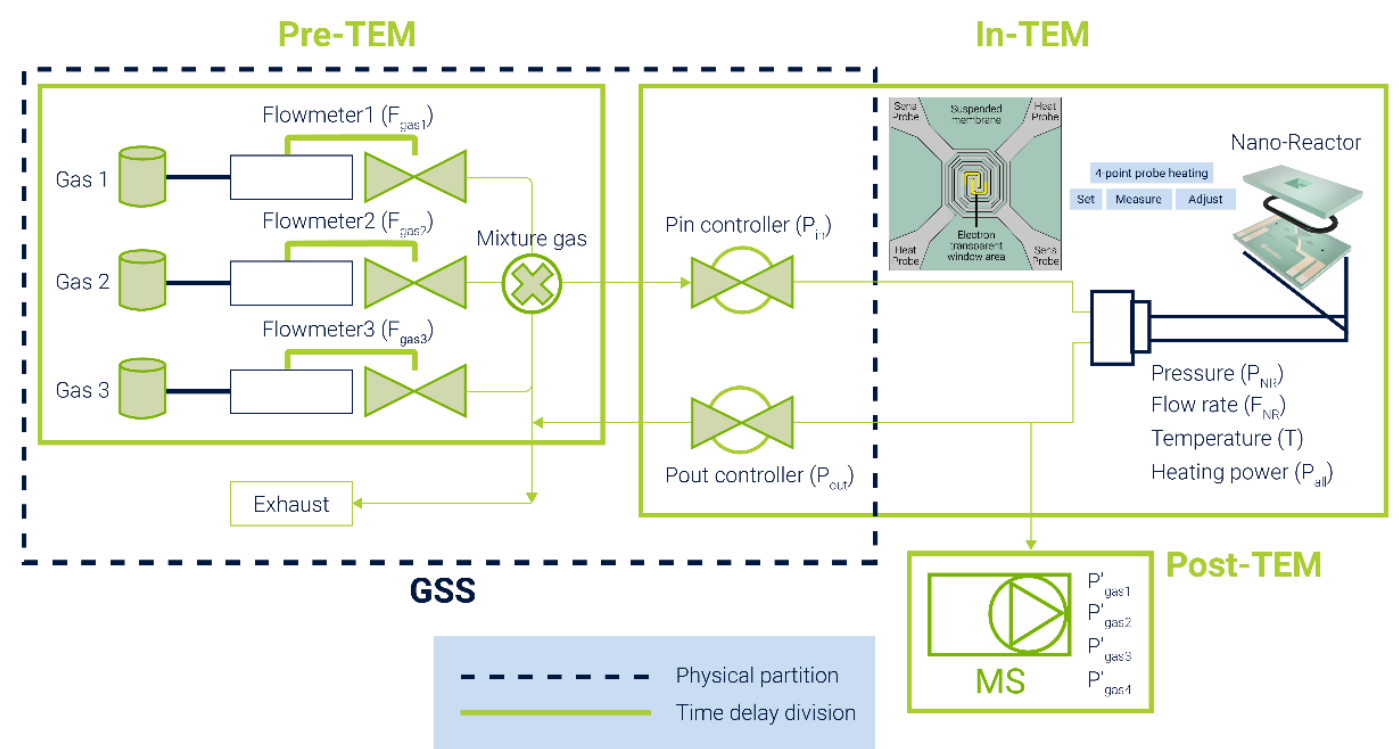

Figure 2. A diagram of the operando gas and heating TEM set-ups

4 Results

\subsection{Calorimetry-based time delay calibration}

Calorimetry is a technique to measure the heat transfer involved in physical or chemical process. In classical calorimetry, the heat is exchanged with a calorimeter calibrated object (26-28). The MEMS-based Nano-Reactor that is being used in this work relies on the same principle. Instead of having a fully insulated object, of which the temperature change is being measured to calculate the heat exchange, the object can 
be kept at an extremely stable constant temperature. In the latter scenario, which is also our case the heat exchange is directly measured by the power consumption differences required to maintain this constant temperature. In our case, this object is the metal microheater inside the Nano-Reactor. The heating power measured by the microheater is a direct measure for the amount of heat transferred by the process under study. It is important to consider there can be more factors playing a role in the heat transfer, which should not be confused. The processes inside the Nano-Reactor involving heat transfer include the microheater itself, sample, gas potential temperature changes of the surroundings to which the heat is lost, endo- or exo-thermic reactions between the sample and gas, and lastly, electron beam radiation. That is to say

$P_{\text {all }}=\left(C_{\text {heater }}+C_{\text {sample }}+C_{\text {gas }}+C_{\text {other }}\right) * \Delta T+\Delta P_{\text {reaction }}+\Delta P_{\text {e-beam }}$

Here $P_{\text {all }}$ refers to the overall heating power in unit of $\mathrm{mW} . C_{\text {heater }}, C_{\text {sample }}$, $C_{\text {gas }}$ and $C_{\text {other }}$ represent the heat capacities of the micro-heater, the TEM sample, the gas and the surrounding respectively. $\Delta \mathrm{T}$ is the temperature variations in a NanoReactor, introduced by user set temperature value different from room temperature, change of surrounding conditions or chemical reactions. $\Delta P_{\text {reaction }}$ and $\Delta P_{e-\text { beam }}$ reflect the heating power variations caused by the chemical reaction and the electron beam irradiation respectively.

The gas composition, which determines $C_{g a s}$ in the Eq.(3), is the only parameter that has direct measurements in pre- and post-TEM, i.e. flow meter in pre-TEM and MS in post-TEM, as described in section 3. It is therefore the most useful parameter for synchronizing pre-, in- and post-TEM data.

The design of the Nano-Reactor microheater hardware and software uses a closedloop feedback control algorithm, which follows the workflow of 'set', 'measure' and 'adjust'. That is to say, the user sets the desired temperature in the software. The software measures the heater temperature, compares it to the user sets value and adjusts if a mismatch found. Normally a few to a few tens of cycles could be needed to finish this complete process, depending on the severity of the changes of the parameters as given in Eq.(3). In general, one cycle would take milliseconds to a few seconds, depending on the heater materials, heating control unit and software security considerations. The cycle time for the microheater used in this work is $1 / 3$ of a second. This cycle time has proven to be a suitable trade-off between controller stability, response time, and settling time. However, depending on the purpose, faster or slower 
cycle times may be preferred and applied for some specific experiments.

Therefore, under isothermal conditions, a $C_{g a s}$ change caused by a gas composition change will be revealed in a $\mathrm{T}$ and a $\mathrm{P}$ change in $1 / 3$ second with current operando set-ups.

As shown in Figure 3(a) and 3(b), keeping all the other experimental parameters the same, the $\mathrm{O}_{2}$ to $\mathrm{CH}_{4}$ ratio was changed from 50 vol\%: 50 vol\% to 75 vol\%: 25 vol\%. Because $C_{\mathrm{O}_{2}}$ is smaller than $C_{\mathrm{CH}_{4}}$, an increase of $\mathrm{O}_{2}$ by $25 \mathrm{vol} \%$ caused an instant temperature increase. The starting point of this change is 34.9 seconds later than the change revealed by gas composition measurement with flow meter in GSS and 45.2 seconds earlier than the detection of the change in MS. To compensate for this temperature change, an immediate heating power reduction was observed to maintain the set isothermal condition, as shown in Figure 3(d). The starting points were derived by intersecting the horizontal line of a stable status and the tangent line of the starting changing profile. The derivation is shown by the dotted line in Figure 3(b) and 3(c).

Another feature supporting the validation of using calorimetry to calibrate timedelay is the synchronization of the calorimetry settling time (i.e. the time taken from one stable value to the other stable one) and gas composition settling time revealed in MS, i.e. $55 \mathrm{~s}$ in current case shown in Figure 3(c) and 3(d).

The $55 \mathrm{~s}$, instead of $1 \mathrm{~s}$ in the GSS part, is caused by the fundamental gas transport and exchange rules, which have dependence on the internal volume (dead volume) of tubing, valves, controllers and Nano-Reactor that allow the gases to diffuse in them and smear out the sharp transition from the one composition to the other. This switching time can be shorter or longer if different gases or flow rates are applied. Meanwhile, this synchronization also provides possibility to get in-clock gas composition inside a Nano-Reactor by nano-calorimetry with the sensitivity well below $1 \mathrm{vol} \%$ of gas composition. More discussions on this point is covered in section 4.3.

This mutual verification proves that calorimetry in the Nano-Reactor is a valid and reliable tool to calibrate the time delays. The maximum error of this time delay is estimated to be $\pm 1 \mathrm{~s}$, considering that the acquisition time per data point of GSS, calorimetry and MS to be $1 \mathrm{~s}, 1 / 3 \mathrm{~s}$ and $1 \mathrm{~s}$ respectively in general experiments. Similar to the feedback cycle time of $1 / 3 \mathrm{~s}$ for heating control, shorter acquisition time can be applied for GSS and MS (e.g. change from "trigger" to "continuous" acquisition mode).

It is important to remain aware of the differences of time delay between different 
gas path locations (which is only determined by gas advection, i.e. transport by bulk motion) and gas switching time from one stable gas composition to another stable composition (which is governed by both advection and diffusion, i.e. transport by random motion) (29). Their differences will be manifested in section 4.2 and 4.3.
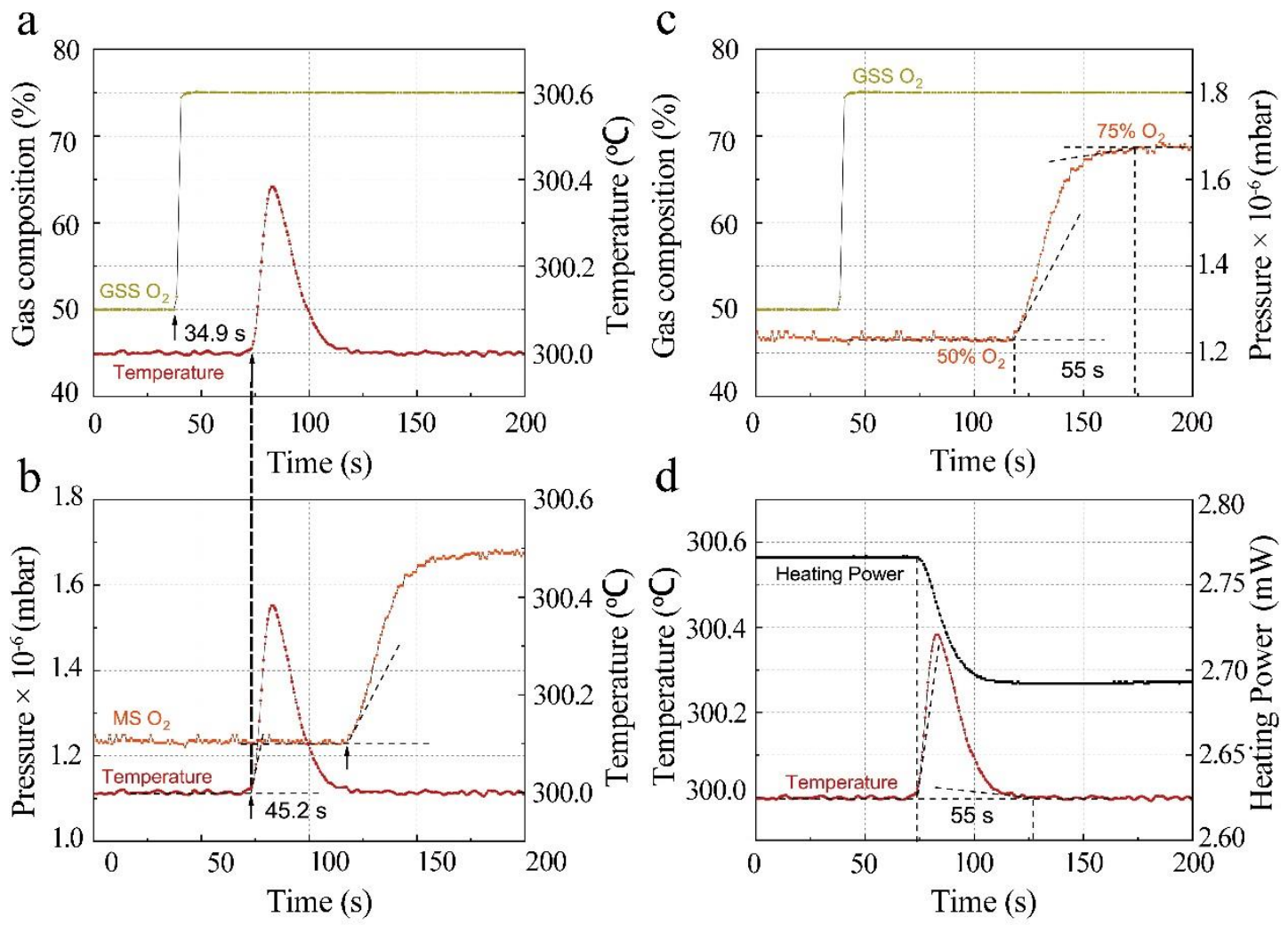

Figure 3. Comparison of parameter changes upon a gas composition change of $\mathrm{O}_{2}$ to $\mathrm{CH}_{4}$ from 50 vol\% : 50 vol\% to 75 vol\% : 25 vol\% (a) $\mathrm{O}_{2}$ ratio measured by GSS flow meter vs Nano-Reactor temperature; (b) $\mathrm{O}_{2}$ partial pressure measured by MS vs NanoReactor temperature; (c) $\mathrm{O}_{2}$ ratio measured by GSS flow meter vs $\mathrm{O}_{2}$ partial pressure measured by MS; (d) Nano-Reactor temperature vs Nano-Reactor heating power.

\subsection{Functional relationship of delayed time with various parameters}

As explained in part 3, we investigated these parameters' influence in the following sections: gas flow rates $\left(\left(F_{N R}\right)\right.$, varied gas composition and gas pressure $\left(P_{N R}\right)$ inside the Nano-Reactor, and the tubing length (L) in connecting the hardware. Maintaining the sum of $F_{\text {gas } 1}, F_{\text {gas } 2}$ and $F_{\text {gas } 3}$ constant, the delayed time between preTEM (GSS) to in-TEM and that of in-TEM to post-TEM (MS) follows the same behavior. Only the results of in-TEM to post-TEM are presented here, while those of pre-TEM to in-TEM are included in the supplementary. 
4.2.1 $F_{N R}$ (gas flow rates inside the Nano-Reactor)

To be concrete, we set isothermal condition of $300{ }^{\circ} \mathrm{C}$ for the measurements in this section. Three or more steps of gas composition change (here $\mathrm{O}_{2}$ and $\mathrm{CH}_{4}$ ) were used to get the time-delay value for one flow rate value. $\mathrm{P}_{\text {in }}$ and $\mathrm{P}_{\text {out }}$ were tuned to maintain the $P_{N R}$ at 900 mbar and vary the $F_{N R}$.

As shown in Figure 4(a), the delay time decreases as the $F_{N R}$ increases. It drops dramatically with $F_{N R}$ from 0.05 to $0.15 \mathrm{mln} / \mathrm{min}$, where the gas transport and exchange happen more likely through diffusion mechanism. It tends to become more flat and linear when $F_{N R}$ is greater than $0.15 \mathrm{mln} / \mathrm{min}$, where gas advection dominates the transport and exchange mechanisms. Here, the ' $\mathrm{mln} / \mathrm{min}$ ' means milliliter per minute normalized to 1 bar pressure. An inversely proportional functional relationship which is an empirical equation between $F_{N R}$ and the time delay $\mathrm{t}$ and covers the whole flow rate range can be fitted to the experimental results (as shown in Figure 4(b)), which can be written as

$$
t=\frac{1}{a+b F_{N R}+c F_{N R}{ }^{2}+d F_{N R}{ }^{3}}
$$

Note, six $F_{N R}$ values of $0.05,0.1,0.2,0.3,0.4$ and $0.5 \mathrm{mln} / \mathrm{min}$ were planned values. More data points nearby were caused by gas composition change modulated $F_{N R}$ variations with fixed $\mathrm{P}_{\mathrm{in}}, \mathrm{P}_{\text {out }}$ and $P_{N R}$ values. As $F_{N R}$ is a reliable experimentally measured (not calculated) value, this variation contributes to more data points for function fitting. 

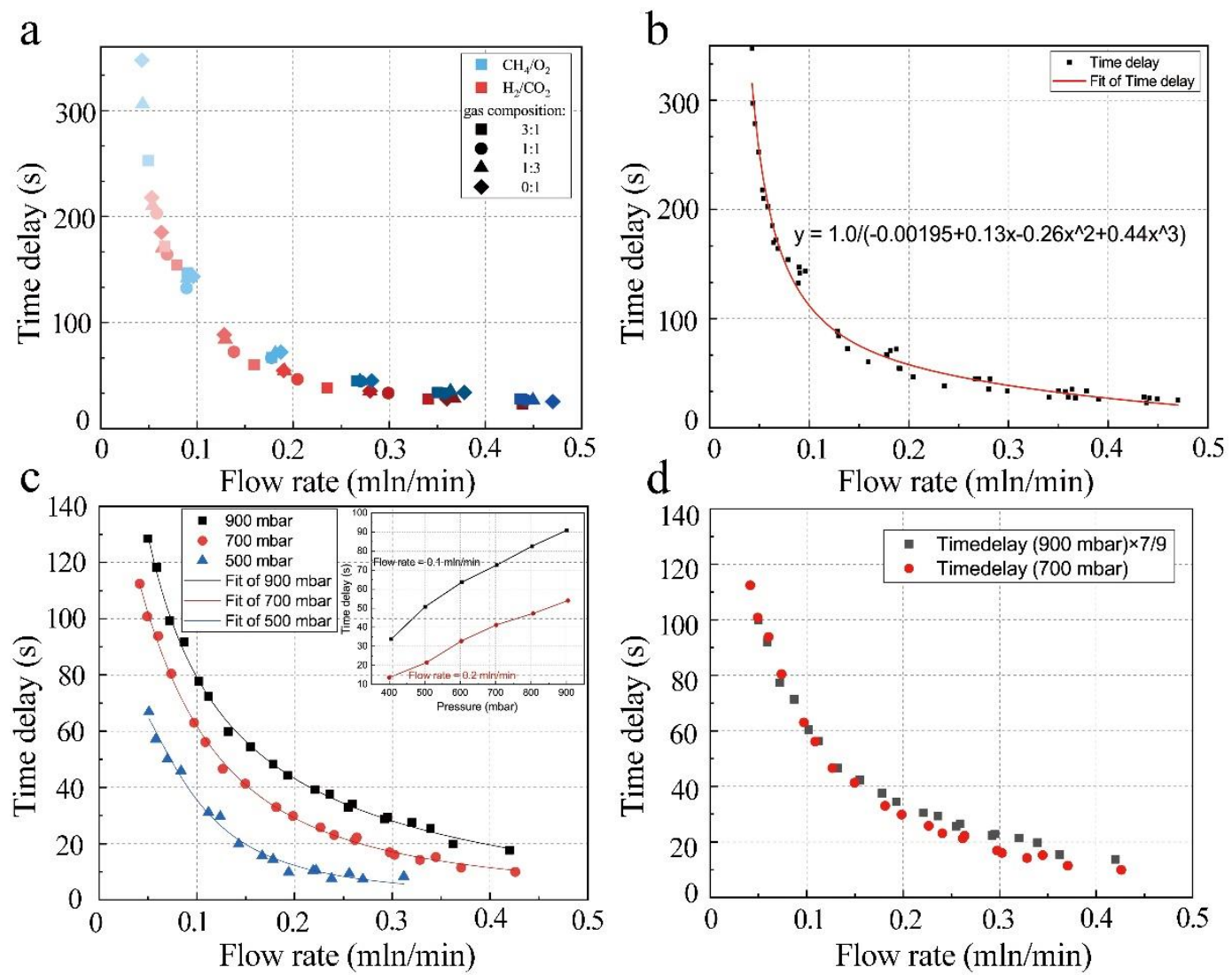

Figure 4. Time delay variation with Nano-Reactor gas flow rate and pressure. (a) Experimental data of delayed time measured with different flow rates and different gas types. The type of mixed gas is $\mathrm{CH}_{4} / \mathrm{O}_{2}$ (blue points) and $\mathrm{H}_{2} / \mathrm{CO}_{2}$ (red points) respectively. The gas composition of $\mathrm{CH}_{4}: \mathrm{O}_{2}$ or $\mathrm{H}_{2}: \mathrm{CO}_{2}$ is changed from 1:0 to 3:1(घ), 3:1 to $1: 1(\bullet), 1: 1$ to $1: 3(\boldsymbol{\Delta}), 1: 3$ to $0: 1(\bullet)$. (b) The fitted function relationship between time delay and $F_{N R}$. (c) Function fitted and experimental time delay data measured under different Nano-Reactor pressure. The inset shows the variation of the time delay with the gas pressure at the same flow rate $(0.1$ and $0.2 \mathrm{mln} / \mathrm{min}$ repsectively). (d) Linear comparison of the delayed time between $P_{N R}$ of 700 mbar and 900 mbar.

\subsection{2 $P_{N R_{-}}$(gas pressure inside Nano-Reactor)}

Same to $F_{N R}$ part, we set isothermal condition of $300{ }^{\circ} \mathrm{C}$ for the measurements in this section. The measurements in section 4.2.1 are repeated for different Nano-Reactor pressures $\left(P_{N R}\right)$, i.e. 500, 700, 900 mbar respectively.

As shown in Figure 4(c), the time delay gradually decreases with the increase of the flow rate at different pressures, all of which can be fitted to the function in Eq.(4) with different fit parameters. When the $F_{N R}$ were controlled to be same $(0.1 \mathrm{mln} / \mathrm{min}$ 
and $0.2 \mathrm{mln} / \mathrm{min}$ ), it is found that the time delay has a linear relationship with the $P_{N R}$ at higher pressure range, while deviating slightly from linear relationship at lower side, as shown in Figure 4(c) inset. Based on this observation, a linear comparison of delayed time covering range of flow rate under 700 mbar and 900 mbar is done and shown in Figure 4(d). It shows very nice agreement at lower flow rates, while slight deviation at higher flow rates.

The reason for the linear matches is that when the volume flow rate in the NanoReactor_ $\left(F_{N R}\right)$ is kept constant, the linear flow rate of the gas in the pipeline will vary due to the change of the gas pressure inside Nano-Reactor $\left(P_{N R}\right)$. According to the Ideal Gas Law:

$\mathrm{PV}=\mathrm{nRT}(5)$

As $F_{N R}$ is normalized to 1 bar pressure, n remains the same when the $F_{N R}$ is consist. When the $P_{N R}$ decreases, the gas volume increases accordingly. In order to keep the volume flow rate constant, the linear flow rate of the gas in the pipeline increases, thereby reducing the time delay. Therefore, the time delay has a linear relationship with the $P_{N R}$.

The slight deviation from linear relationship at higher flow rates and lower pressure could be attributed to detailed gas transportation mechanisms, which needs further investigations. Therefore, a good estimation of the time delay under different $P_{N R}$ can be derived from one $P_{N R}$ measurements through a linear factor. Time delay measurements for fine steps of $P_{N R}$ values are suggested for more precise values.

\subsubsection{Gas types}

In section 4.1, 4.2.1 and 4.2.2, $\mathrm{CH}_{4}$ and $\mathrm{O}_{2}$ are used. The selection of gas types there is arbitrary. To study gas types' influence, $\mathrm{H}_{2}$ and $\mathrm{CO}_{2}$ are used as alternative and comparative gases to repeat the measurements in section 4.1.1. As shown in Figure 4(a), the time delay values measured with $\mathrm{H}_{2}$ and $\mathrm{CO}_{2}$ can be fitted with the same function of $\mathrm{CH}_{4}$ and $\mathrm{O}_{2}$. Small deviation of 1 or $2 \mathrm{~s}$ for flow rate around $0.2 \mathrm{mln} / \mathrm{min}$ are ignorable experimental errors, as confirmed by repetitive measurements with other gases. That is to say, the delayed time has no dependence on the gas types.

\subsubsection{Connecting Tubing length (L)}

The whole gas path length consists of length of gas path in GSS, in connecting tubing, in TEM holder and inside MS. Theoretically, the travel time (i.e. the delayed time in current scenario) is proportional to the whole gas path length between any two locations. More complex, rather than linear, monotonic relationship can be expected as 
rendered by gas convection and diffusion mechanisms. It is obvious that the connecting tubing length, the only part a user can measure and change, is only one part of the whole gas path. So a detailed functional relationship between connecting gas tubing length and delayed time is not studied.

However, a general suggestion of using as short as possible connecting tubing can be made for shorter time delays in experiments.

\subsection{Gas switching time and in-clock composition synchronization}

Similarly, we investigated various parameters' influence on the gas switching time. As shown in Figure 5(a) and 5(b), the switching time also decrease with flow rates increases, but doesn't follow the same function in Eq.(3) and has differences between $\mathrm{CH}_{4}: \mathrm{O}_{2}$ and $\mathrm{H}_{2}: \mathrm{CO}_{2}$ cases.

As briefly mentioned in section 4.1, the gas switching time needed to change from one gas composition to another gas composition is governed by both gas advection and diffusion.

If advection dominates, the incoming gas simply pushes out the gas that was initially present in the Nano-Reactor. In this case, the mathematics are straightforward; integration over the flow path in the gas path divided by the local gas flow speed. Estimation of this time can be done with gas path length inside the Nano-Reactor of 5 $\mathrm{mm}$, tubing diameter of $250 \mu \mathrm{m}$, flow speed of $0.5 \mathrm{mln} / \mathrm{min}$, which results in a switching time of $0.0294 \mathrm{~s}$.

If diffusion dominates, the diffusive flux of gases is governed by the diffusion coefficient $\mathrm{D}$ and the concentration gradient $(\nabla c)$, as given by Fick's first law:

$$
\vec{J}=-D \nabla c(6)
$$

The diffusion coefficient $\mathrm{D}$ is influenced by the gas composition, temperature, and pressure. Detailed explanations can be found in these literatures $(29,30)$ which is beyond the scope of the current manuscript.

As shown in the Figure 5, the switching time is related to the inter-diffusion coefficient of one gas in another and varies with gas types significantly. The switching time of hydrogen (to other gases, e.g. $\mathrm{CO}_{2}$ in Figure 5(b)) in the Nano-Reactor is much longer than other gases. Then $\mathrm{CH}_{4}, \mathrm{O}_{2}, \mathrm{CO}_{2}$ can be sorted from longer to shorter switching time under same flow rate. This is because that gases with lighter molecules have higher diffusion coefficients and thus higher chance of diffusion against flow direction. 

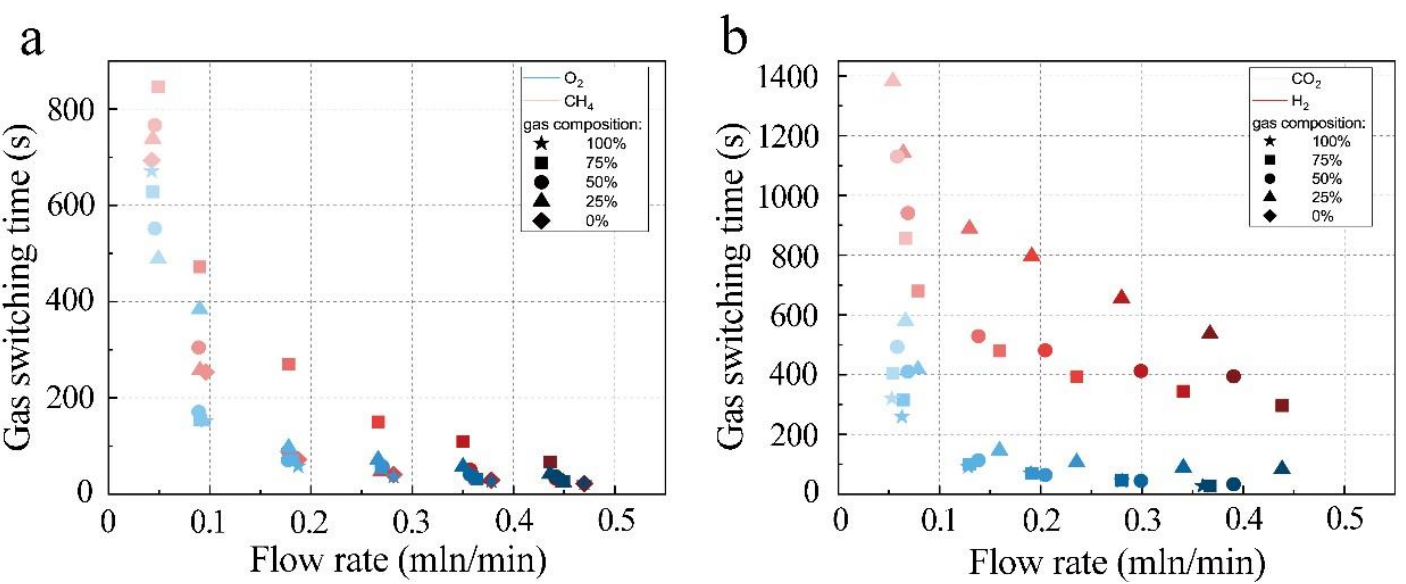

Figure 5. Gas switching time varies with gas flow rate. (a) The gas switching time of $\mathrm{O}_{2}$ (i.e. decrease $\mathrm{O}_{2}$ and increase $\mathrm{CH}_{4}$ ratio, blue points) and $\mathrm{CH}_{4}$ (i.e. decrease_- $\mathrm{CH}_{4}$ and increase $\mathrm{O}_{2}$ ratio, red points) in their mixed gas changes with the $F_{N R}$. (b) The gas switching time of $\mathrm{CO}_{2}$ (i.e. decrease $\mathrm{CO}_{2}$ and increase $\mathrm{H}_{2}$ ratio, blue points) and $\mathrm{H}_{2}$ (i.e. decrease $\mathrm{H}_{2}$ and increase $\mathrm{CO}_{2}$ ratio, red points) in their mixed gas changes with the $F_{N R}$. The $F_{N R}$ is controlled at $0.05,0.1,0.2,0.3,0.4,0.5 \mathrm{mln} / \mathrm{min}$ by $\mathrm{P}_{\text {in }}$ and $\mathrm{P}_{\text {out }}$. Then the gas composition of $\mathrm{CH}_{4}: \mathrm{O}_{2}$ or $\mathrm{H}_{2}: \mathrm{CO}_{2}$ is changed from 1:0 to 3:1, 3:1 to 1:1, 1:1 to 1:3, 1:3 to $0: 1$, so the gas composition after switching in the Nano-Reactor are $0_{-}$vol.\%( $\%$,

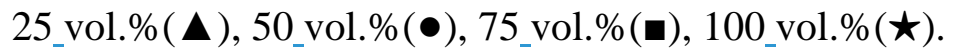

\subsection{Summarize}

In short, the delayed time between different parts, either group in GSS, TEM, MS, or group in pre-TEM, in-TEM and post-TEM, is determined by the gas flow rate in the Nano-Reactor $\left(F_{N R}\right)$, the gas pressure inside the Nano-Reactor $\left(P_{N R}\right)$ and total gas path length. As people normally don't change the connecting tubing length in one lab for one operando TEM set-up and the $P_{N R}$ is always consist during a Operando TEM experiment, the founded function relation between $F_{N R}$ and delayed time can be used for automatic time delay calibration. If $P_{N R}$ varies, a linear factorization to one pressure measurements can be applied to get good estimation for different pressures and a precise value can be got by functional relationships fitted for different pressure values.

The gas switching time (or say settling time) is governed by more complicate intercorrelations among various parameters. It is difficult to build simple functional relationship. However, an in-clock synchronization to gas composition is achievable and useful for the operando research.

\section{Automation}




\subsection{Time calibration (delay correction) script:}

Based on the above results, an open source python script (https://github.com/DENSsolutions/Climate-time-delay-characterization-andcalibration) incorporating the fitted function has been written for calibrating the time delays in practical experiments.

Practical experiments, compared to the simplified situation only involving gas composition change, can easily be complicated by chemical reactions. For example, under fixed inlet and outlet pressure control mode, i.e. fixed pressure control mode, a reaction could cause a Nano-Reactor gas composition change and thus flow rate change. It is difficult and potentially un-reliable to use one time-delay value for the whole experiment. Therefore, an automatic calibration for real experimental data is essential.

To realize the automation, the time calibration script first splits the system parameters into the three different sets: Pre-TEM, In-TEM and Post-TEM parameters. Each set of parameters' timestamp is corrected based on the calculated time offset that results from inserting the measured flow rate to the fitted time delay curve.

The script saves the three datasets (Pre-TEM, In-TEM and Post-TEM) individually with their own time resolution, and creates a synchronized log file in which the Pre-TEM and Post-TEM parameters are interpolated towards the In-TEM dataset timestamps. These data can then be used to synchronize with TEM images/spectra to achieve whole operando gas and heating TEM data synchronization.

\subsection{Time delay curve characterization script:}

Furthermore, based on the upper results, a time delay curve characterization script that automates the functional relationship derivation has been developed and shared as open source code (https://github.com/DENSsolutions/Climate-time-delaycharacterization-and-calibration).

The script utilizes the DENSsolutions Impulse API and various Python library to control and retrieve measurements from the GSS, heating control unit and MS. As shown in Figure 6(a), these parameters, as well as the rolling averages of the signals are monitored.

The script performs a defined amount of measurements at a series of different flow rates. Both the number of measurements (i.e. gas composition change steps) at each flow rate and the number of flow rate values can be adjusted to optimize between speed and precision. 
For each measurement, after a stable flow rate and stable gas composition are achieved, noise measurements which is the absolute change value for the signals (Figure 6(b)) are performed for the heating power (In-TEM) and the MS measured gas partial pressure (Post-TEM) to determine the thresholds to detect the changes. Then it toggles between two gas states in GSS while keeping Nano-Reactor pressure the same. The measurements in Pre-TEM are plotted in Figure 6(c) and the timestamp of this change is stored in pre-TEM part. The script then monitors the temperature and the heater power measurement (In-TEM) and the gas partial pressure measurement (PostTEM) for a change using the earlier defined thresholds. When a threshold is exceeded, the start of the change is determined, and the changes of the signals in In-TEM (Figure 6(d)) and Post-TEM (Figure 6(e)) will be plotted to monitor the accuracy of the detection and the corresponding timestamps are stored in the in-TEM and post-TEM parts respectively. These three timestamps will be used to calculate the delayed time, and plotted against the average flow rate in Figure 6(f \& g).

This process is repeated for each flow rate. The resulting offset times are stored along with the corresponding flow rates inside one data frame. After all the measurements are done, curves are fitted to the data using the optimize function from the SciPy module, as shown in Figure 6(f \& g). The synchronized data corrected by the Time Calibration Script which is loaded with the final fitted time delay parameters is shown in Figure 7.

a

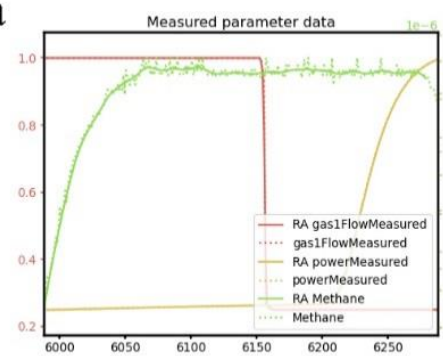

b

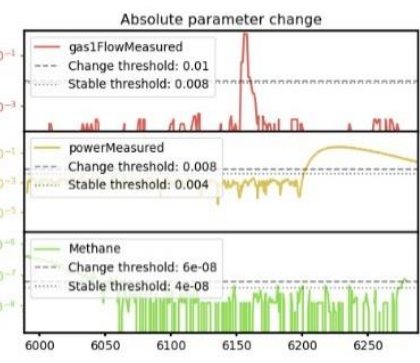

Test 15/15 status: Last test finished.
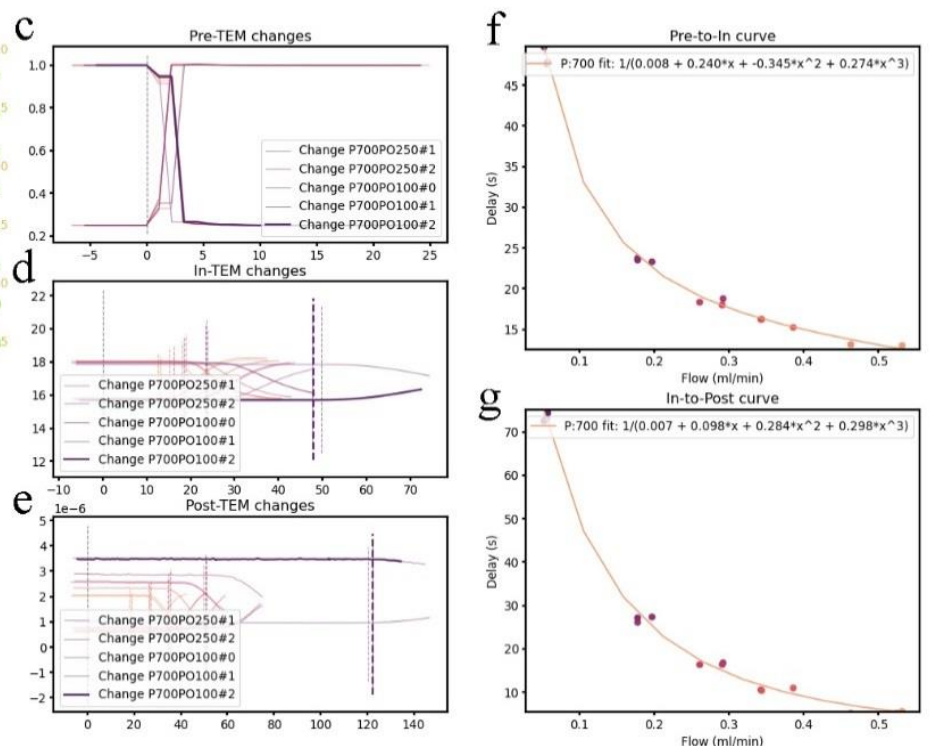

Figure 6. The automatic test results of time delay using the Time Delay Curve Characterization Script. (a) The signals and its rolling average of measured flow rate of 
gas1, heating power, and mass spectrometer signal of gas1. (b) The absolute change values for the signals are monitored to detect the changing time. (c-e) The changes of the signal in Pre-TEM (c), In-TEM (d) and Post-TEM (e) will be plotted after this signal crosses the lower threshold for the changing state. (f-g) The time delays between the pre-TEM and in-TEM_f) and between in-TEM and post-TEM_(g) are calculated, and the functional relationship can be fitted.

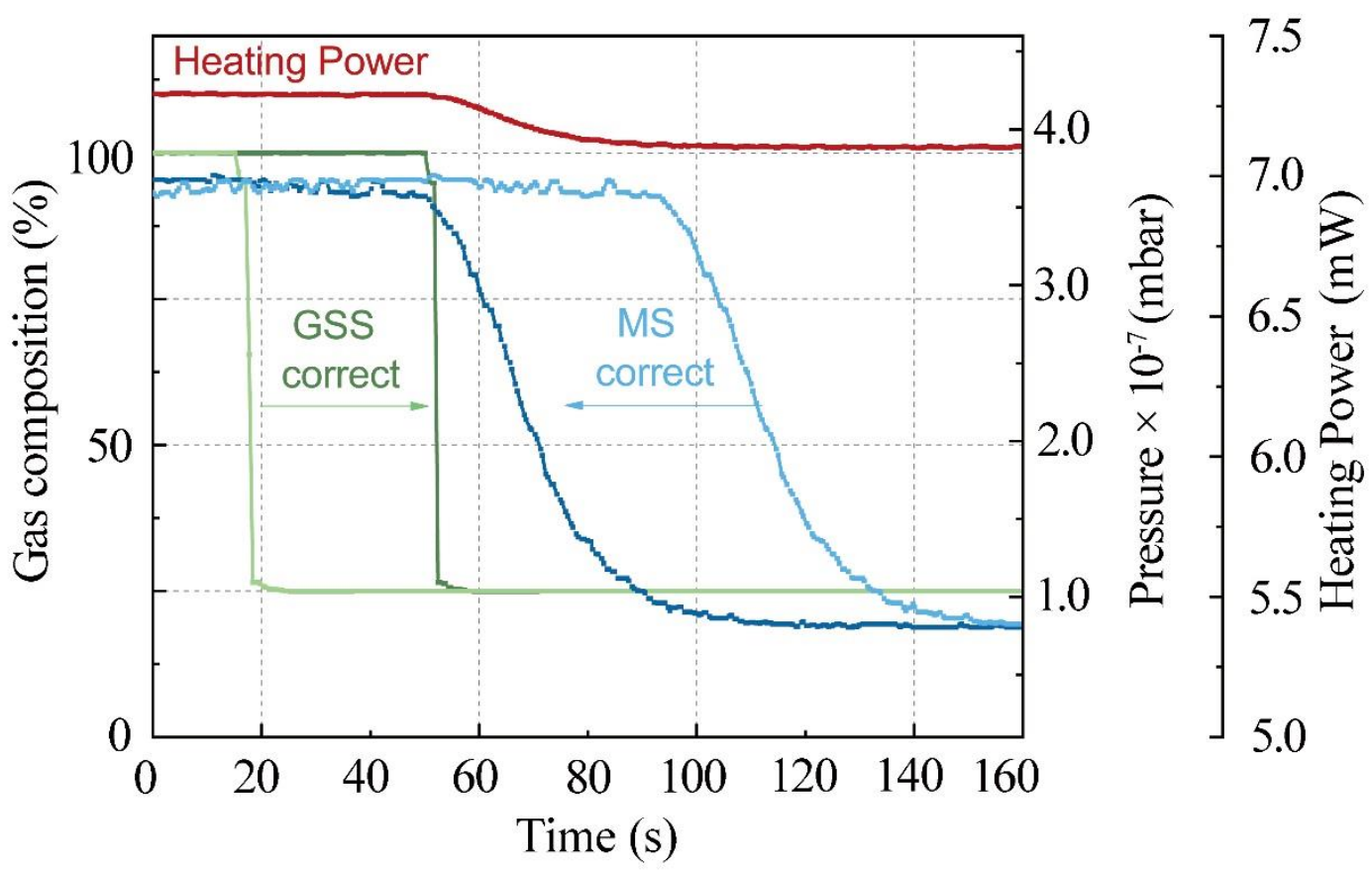

Figure 7. The synchronized data corrected by the Time Delay Correction scripts.

\section{Operation protocol}

With the above conclusions and scripts, a conductive guidance to calibrate timedelays in operando gas and heating TEM is given here.

1 Set-up essential hardware, including gas supply system, e.g. DENSsolutions Climate GSS in the current work, a mass spectrometer, two gases with different heat capacities, e.g. $\mathrm{CH}_{4}$ and $\mathrm{O}_{2}$, a sample holder assembled with an empty Nano-Reactor and pass the leak test.

2 Insert the holder into the vacuum test tube of the Gas Supply System (mimicking TEM vacuum condition) or TEM. Connect all the essential connections.

3 Open control software, starting gas flow at a pressure, and set a temperature beyond room temperature (system will give heating power). Choose the $\mathrm{P}$ vs $\mathrm{t}$ mode of MS to detect the composition of the gas. 
4 Start the Time Delay Curve characterization Script, it will take time delay measurements at different flow_rates automatically. When all measurements are completed, the final fitted time delay curve parameters are saved in a calibration file.

5 Load the calibration file into the Time Delay Correction scripts to remove the time-delays in more realistic and complicated experimental data after acquisition. The fitted function and Time Delay Correction script can also be applied in real-time data acquisition to enable synchronized data visualization during experiments.

\section{Conclusion}

To conclude, operando gas and heating TEM is powerful in correlating material process (e.g. gas and thermal environment here), structure and property (e.g. precursor conversion) to optimize materials design. Its capabilities also manifest its complexities in data synchronization and interpretation. In this work, we have shown the importance and the guidance to calibrate the time delays involved in operando gas and heating TEM for reliable multi-factor information correlations. With detailed understanding of the experimental set-ups, critical parameters, including gas pressure $\left(P_{N R}\right)$ and gas flow rate $\left(F_{N R}\right)$, gas types $\left(\mathrm{C}_{\mathrm{gas}}\right)$, connecting tubing length $(\mathrm{L})$, are sorted out. Investigations to their influences on the delayed time led to the conclusion that a function relationship (shown in Eq.(4)) between delayed time and $F_{N R}$ can be used to manually and automatically calibrate the time delays. Although the length of gas switching has more complicated correlations to various parameters, their changing profile revealed in nanocalorimetry can be used to get in-clock average gas composition in the sample area. Furthermore, with the current research results and conclusions, open source scripts for automatic characterizing and removing the time delay in operando gas and heating TEM can feasibly speed up the valid data synchronization and ensure more reliable information correlations.

\section{Acknowledgement}

This work received financial support from the DICP-PRI Joint Foundation (PRIKY18015); National Natural Science Foundation of China (22072150); CAS Youth Innovation Promotion Association (2019190); DICP-DENS Laboratory for In Situ Microscopy Applications Project. Financial support by the European Union's HORIZON 2020 Research and Innovation Programme ESTEEM3 under grant agreement No. 823717 is gratefully acknowledged. 


\section{Competing interests}

The authors declare that they have no competing interests.

References:

1. D. S. Su, B. S. Zhang, R. Schlogl, Electron Microscopy of Solid Catalysts-Transforming from a Challenge to a Toolbox. Chem Rev 115, (2015) 2818-2882. https://doi.org/10.1021/cr500084c.

2. F. Tao, P. A. Crozier, Atomic-Scale Observations of Catalyst Structures under Reaction Conditions and during Catalysis. Chem Rev 116, (2016) 3487-3539. https://doi.org/10.1021/cr5002657.

3. E. D. Boyes, A. P. LaGrow, M. R. Ward, T. E. Martin, P. L. Gai, Visualizing single atom dynamics in heterogeneous catalysis using analytical in situ environmental scanning transmission electron microscopy. Philos $T R$ Soc A 378, (2020) https://doi.org/10.1098/rsta.2019.0605.

4. P. L. Hansen et al., Atom-resolved imaging of dynamic shape changes in supported copper nanocrystals. Science 295, (2002) 2053-2055. DOI: 10.1126/science.1069325.5. K. F. Kalz et al., Future Challenges in Heterogeneous Catalysis: Understanding Catalysts under Dynamic Reaction Conditions. Chemcatchem 9, (2017) 17-29. https://doi.org/10.1002/cctc.201600996.

6. X. B. Zhang et al., Reversible loss of core-shell structure for $\mathrm{Ni}-\mathrm{Au}$ bimetallic nanoparticles during $\mathrm{CO}_{2}$ hydrogenation. Nat Catal 3, (2020) 411-417. https://doi.org/10.1038/s41929-020-0440-2.

7. E. D. Boyes, P. L. Gai, Environmental high resolution electron microscopy and applications to chemical science. Ultramicroscopy 67, (1997) 219-232. https://doi.org/10.1016/S0304-3991(96)00099-X.

8. J. F. Creemer et al., Atomic-scale electron microscopy at ambient pressure. Ultramicroscopy 108, (2008) 993-998. https://doi.org/10.1016/j.ultramic.2008.04.014.

9. W. T. Yuan et al., Direct In Situ TEM Visualization and Insight into the Facet-Dependent Sintering Behaviors of Gold on $\mathrm{TiO}_{2}$. Angew. Chem.Int. Edit. 57, (2018) 16827-16831. https://doi.org/10.1002/anie.201811933.

10. W. T. Yuan et al., Visualizing $\mathrm{H}_{2} \mathrm{O}$ molecules reacting at $\mathrm{TiO}_{2}$ active sites with transmission electron microscopy. Science 367, (2020) 428-430. DOI: $10.1126 /$ science.aay2474.

11. L. Q. Zhang et al., Lithium whisker growth and stress generation in an in situ atomic force microscope-environmental transmission electron microscope set-up. Nat Nanotechnol 15, (2020) 94-98. https://doi.org/10.1038/s41565-019-0604-x.

12. H. Yoshida et al., Visualizing Gas Molecules Interacting with Supported Nanoparticulate Catalysts at Reaction Conditions. Science 335, (2012) 317-319. DOI: 10.1126/science.1213194. 
13. P. A. Crozier, S. Chenna, In situ analysis of gas composition by electron energy-loss spectroscopy for environmental transmission electron microscopy. Ultramicroscopy 111, (2011) 177-185. https://doi.org/10.1016/j.ultramic.2010.11.005.

14. S. Chenna, P. A. Crozier, Operando Transmission Electron Microscopy: A Technique for Detection of Catalysis Using Electron Energy-Loss Spectroscopy in the Transmission Electron Microscope. Acs Catal 2, (2012) 2395-2402. https://doi.org/10.1021/cs3004853.

15. B. K. Miller, P. A. Crozier, Analysis of Catalytic Gas Products Using Electron Energy-Loss Spectroscopy and Residual Gas Analysis for Operando Transmission Electron Microscopy. Microsc Microanal 20, (2014) 815-824. https://doi.org/10.1017/S1431927614000749.

16. M. Plodinec et al., Versatile Homebuilt Gas Feed and Analysis System for Operando TEM of Catalysts at Work. Microsc Microanal 26, (2020) 220-228. https://doi.org/10.1017/S143192762000015X.

17. S. W. Chee, J. M. Arce-Ramos, W. Q. Li, A. Genest, U. Mirsaidov, Structural changes in noble metal nanoparticles during $\mathrm{CO}$ oxidation and their impact on catalyst activity. Nat. Commun. 11, (2020). https://doi.org/10.1038/s41467-020-16027-9.

18. I. Persson et al., How Much Oxygen Can a MXene Surface Take Before It Breaks? Adv Funct Mater 30, (2020) https://doi.org/10.1002/adfm.201909005.

19. S. B. Vendelbo et al., Visualization of oscillatory behaviour of $\mathrm{Pt}$ nanoparticles catalysing CO oxidation. Nat. Mater. 13, (2014) 884-890. https://doi.org/10.1038/nmat4033.

20. S. F. Tan et al., Real-Time Imaging of Nanoscale Redox Reactions over Bimetallic Nanoparticles. Adv Funct Mater 29, (2019). https://doi.org/10.1002/adfm.201903242.

21. M. Plodinec, H. C. Nerl, F. Girgsdies, R. Schlogl, T. Lunkenbein, Insights into Chemical Dynamics and Their Impact on the Reactivity of Pt Nanoparticles during CO Oxidation by Operando TEM. Acs Catal 10, (2020) 3183-3193. https://doi.org/10.1021/acscatal.9b03692.

22. H. H. Perez-Garza et al., The "Climate" System: Nano-Reactor for in-situ analysis of solid-gas interactions inside the TEM. Int Conf Nano Micro, (2016) 85-90. doi: 10.1109/NEMS.2016.7758206.

23. Zhou, D., Spruit, R., Pen, M., Garza, H., \& Xu, Q. Correlative in-situ gas and heating TEM: integrating calorimetry and mass spectroscopy. Microsc Microanal 26(S2), (2020) 3044-3046. doi:10.1017/S1431927620023636.

24. N. T. Nguyen, Micromachined flow sensors - a review. Flow Meas Instrum 8, (1997) 7-16. https://doi.org/10.1016/S0955-5986(97)00019-8.

25. J. H. Gross, Mass spectrometry: a textbook. Springer International Publishing, Cham, 2017. doi: 10.1007/978-3-642-10711-5.

26. S. L. Lai, G. Ramanath, L. H. Allen, P. Infante, Z. Ma, High-Speed (104 
$\left.{ }^{\circ} \mathrm{C} / \mathrm{S}\right)$ Scanning Microcalorimetry with Monolayer Sensitivity $\left(\mathrm{J} / \mathrm{M}^{2}\right)$. Appl Phys Lett 67, (1995) 1229-1231. https://doi.org/10.1063/1.115016.

27. A. E. Lopeandia, J. Valenzuela, J. Rodriguez-Viejo, Power compensated thin film calorimetry at fast heating rates. Sensor Actuat a-Phys 143, (2008) 256-264. https://doi.org/10.1016/j.sna.2007.11.006.

28. M. D. Grapes et al., Combining nanocalorimetry and dynamic transmission electron microscopy for in situ characterization of materials processes under rapid heating and cooling. Rev Sci Instrum 85, (2014). https://doi.org/10.1063/1.4892537.

29. R. Spruit, Delft University of Technology, Delft (2017). http://resolver.tudelft.nl/uuid:7749f4ff-6a05-41b2-b82c-7b763788f36c.

30. I. L. Mostinsky, "DIFFUSION COEFFICIENT," in A-to-Z Guide to Thermodynamics, Heat and Mass Transfer, and Fluids Engineering. Begellhouse, (2011). doi:10.1615/AtoZ.d.diffusion_coefficient. 